\title{
Erratum to: An elastic-plastic contact model for line contact structures [Sci. China-Phys. Mech. Astron. 61, 054611 (2018)]
}

\author{
HaiBin Zhu ${ }^{1,2}$, YingTao Zhao ${ }^{1}$, ZhiFeng $\mathrm{He}^{1}$, RuiNan Zhang ${ }^{3}$, and ShaoPeng $\mathrm{Ma}^{{ }^{*}}$ \\ ${ }^{1}$ School of Aerospace Engineering, Beijing Institute of Technology, Beijing 100081, China; \\ ${ }^{2}$ State Key Laboratory of Explosion Science and Technology, Beijing institute of Technology, Beijing 100081, China; \\ ${ }^{3}$ National Engineering Research Centre of Surveying and Mapping, Beijing 100039, China
}

Received April 26, 2018; accepted April 26, 2018; published online June 20, 2018

Erratum to: Sci. China-Phys. Mech. Astron. 61, 054611 (2018), https://doi.org/10.1007/s11433-017-9146-9

Citation: H. B. Zhu, Y. T. Zhao, Z. F. He, R. N. Zhang, and S. P. Ma, Erratum to: An elastic-plastic contact model for line contact structures [Sci. China-Phys. Mech. Astron. 61, 054611 (2018)], Sci. China-Phys. Mech. Astron. 61, 084651 (2018), https://doi.org/10.1007/s11433-018-9235-3

In the original publication [1] of this paper, eq. (14) should be written as:

$$
\begin{aligned}
& \sigma_{\text {ave }} \\
& =\left\{\begin{array}{cc}
\frac{\pi E^{*} \varepsilon^{*}}{8}, & \varepsilon^{*}<\frac{3.80 \sigma_{\mathrm{s}}}{E^{*}}, \\
\frac{\pi E^{*} \varepsilon^{*}}{8}+0.95\left(1-\frac{H^{*}}{E^{*}}\right) \sigma_{\mathrm{s} \sqrt{1-\left(\frac{3.80 \sigma_{\mathrm{s}}}{E^{*} \varepsilon^{*}}\right)^{2}}} \\
-\frac{\left(E^{*}-H^{*}\right) \varepsilon^{*}}{4} \times \arcsin \sqrt{1-\left(\frac{3.80 \sigma_{\mathrm{s}}}{E^{*} \varepsilon^{*}}\right)^{2}}, & \varepsilon^{*} \geq \frac{3.80 \sigma_{\mathrm{s}}}{E^{*}},
\end{array}\right.
\end{aligned}
$$

where " 0.85 " in the original equation should be " 0.95 ".

1 H. Zhu, Y. Zhao, Z. He, R. Zhang, and S. Ma, Sci. China-Phys. Mech. Astron. 61, 054611 (2018).

The online version of the original article can be found at https://doi.org/10.1007/s11433-017-9146-9 